\title{
WORLD UNION OF JEWISH STUDENTS (WUJS) UNION MONDIALE DES ETUDIANTS JUIFS (UMEJ)
}

P.O. Box 7914

91077 Jerusalem (Israel)

1924

Tel: (2) 637482

Fax: (2) 224153

Telex: 25615 (att. wujs)

Executive Director: Daniel Yossef

The aims of the Union are: to unite the national association of Jewish students in all countries; to represent Jewish students in academic life; to further and protect Jewish student interest and to ensure adequate representation of their opinion at meetings of international and other organizations; to cooperate with any organization which is concerned to promote the interests of students in general; to strengthen the ties of Jewish students with Israel; and to organize activity on behalf of Jewish communities in distress. Cultural guidance has been given to its unions (totalling 200,000 members in 38 countries); International Seminars, Holiday Schools and Camps have been arranged, and consultative status as well as regular contacts both between other national and international bodies have been reinforced. WUJS operates Project AREIVIM, a unique service programme for Diaispora communities.

Apart from its World Secretariat office in Jerusalem, WUJS maintains its regional offices in Brussels, New York, Mexico City, Sydney, Johannesburg, and Tel Aviv.

Principal publications: WUJS Report (monthly); Shofar (quarterly); Fourth World Haggadah; Learning to Learn (a guide to Yeshiva education, in English); Toward
Jewish-Palestinian Reconciliation.

Les buts de l'Union sont: d'unir les associations nationales des étudiants juifs dans tous les pays; de représenter les étudiants juifs dans le monde académique; de servir et protéger les intérêts de l'étudiant juif et $d$ 'assurer une représentation adéquate de leur opinion aux réunions des organisations internationales et autres; de collaborer avec toute organisation qui a pour but de servir les intérêts des étudiants engénéral; de resserrer. les liens des étudiants juifs avec l'Israël; d'organiser des activités en faveur des communautés juives dans la détresse. Une orientation a été donnée à ses unions $(200.000$ membres dans 38 pays); des séminares internationaux, des écoles de vacances et de voyages ont été organisées; des étudiants ont bénéficié de programmes d'échanges et de voyages; et des contacts aussi bien entre les unions affilées elles-mêmes qu'avec d'autres organismes nationaux et internationaux on été renforcés. L'UMEJ administre le Projet AREIVIM, un programme de service pour les communautés juives, unique en son genre.

Outre son Secrétariat international à Jerusalem, l'UMEJ compte des bureaux régionaux à Bruxelles, New' York, Mexico, Sydney, Johannesbourg, et Tel Aviv.

Publications principales: "Rapport UMEJ" (mensuel); "Shofar" (trimestriel, également en français); "Fourth World Haggadah"; "Learning to Learn" (un guide de l'éducation Yeshiva, en anglais); "Tow'ard Jewish-Palestinian Reconciliation". 